\title{
Um Estudo Exploratório do Cooja para Simulação de Cenário em Internet das Coisas
}

\author{
Dienefer Fialho dos Santos ${ }^{1}$, Fábio Paulo Basso ${ }^{1,2}$, Marcelo Caggiani Luizelli ${ }^{1,2}$, \\ Saimon Martins Cabrera ${ }^{1}$ \\ ${ }^{1}$ Universidade Federal do Pampa (UNIPAMPA) \\ ${ }^{2}$ Programa de Pós-Graduação em Engenharia de Software (PPGES) \\ Av. Tiarajú, 810 - Bairro: Ibirapuitã - Alegrete - RS - CEP: 97546-550 \\ \{dienefersantos.aluno, fabiobasso, marceloluizelli, saimoncabrera.aluno\} \\ Qunipampa.edu.br
}

\begin{abstract}
Courses taught using problem-based learning (PBL) have simulations as a valuable asset for exercising the students' problem solving skills. The use of computational simulations have the potential to explore a variety of problems related to Internet of Things without the costs and risks present in physical implementations. This work presents some example problems in the agriculture domain and a simulation using Cooja Simulator that can be used as a starting point for their use with the PBL methodology.
\end{abstract}

Resumo. Disciplinas ministradas com aprendizagem baseada em problemas $(A B P)$ possuem simulações como um recurso valioso para exercitar as habilidades de resolução de problemas dos alunos. O uso de simulações computacionais têm o potencial de explorar uma variedade de problemas relacionados à Internet das Coisas sem os custos e riscos presentes nas implementações físicas. Este trabalho apresenta alguns exemplos de problemas no domínio de agricultura e uma simulação utilizando o Cooja Simulator que podem ser usados como ponto de partida para o seu uso com a metodologia ABP.

\section{Introdução}

Segundo previsões da Organização das Nações Unidas (ONU), até 2050 a população mundial vai chegar a 9.7 bilhões de pessoas, o que cria uma necessidade de aumento de produção de alimentos. O aumento da expectativa de vida e a urbanização global também gera demanda de uma maior variedade de alimentos. Isso exige que a produção agrícola não só aumente como se torne mais eficiente. [ONU 2021]

Wireless Sensor Networks são redes compostas por nodos sensores que auxiliam no monitoramento de áreas nas quais são colocados. As redes podem ser estruturadas ou não estruturadas e o número de sensores pode chegar aos milhares. Wireless Sensor Networks estruturadas são aquelas nas quais a implantação dos sensores é feita em locais precisos e oferecem fácil acesso aos sensores para manutenção. De forma oposta, Wireless Sensor Networks não estruturadas são normalmente utilizadas em áreas de difícil acesso, os sensores são implantados em grande quantidade sem seguir uma arquitetura tão precisa quanto nas estruturadas. [Thakur et al. 2019] 
Neste contexto, a modelagem e implementação de Wireless Sensor Networks enfrenta desafios, não só pela limitação dos dispositivos utilizados, como também a dificuldade de testar as soluções de forma satisfatória antes da implantação. Simulações, por outro lado, permitem a realização de testes próximos do ambiente real sem exigir dispositivos físicos [Khan et al. 2016].

No estudo de [Abuarqoub et al. 2016], os autores defendem que o sucesso da implementação de uma WSN depende de se ter os conceitos propostos devidamente testados e avaliados antes de partir para uma plataforma física. Simulações oferecem uma alternativa eficiente e de baixo custo para analisar a performance da aplicação antes da implantação, especialmente considerando que testbeds de grande porte não são viáveis devido ao custo e complexidade. Apesar de ser possível acessar algumas testbeds gratuitamente, elas possuem uma maior complexidade, não sendo adequadas para estudantes que estão apenas iniciando sua pesquisa na área.

O curso de Engenharia de Software na Universidade Federal do Pampa (UNIPAMPA) possui disciplinas de Resolução de Problemas (RP), esses componentes curriculares tem como base a metodologia de Aprendizagem Baseada em Problemas (ABP). Essa metodologia surge da necessidade de realizar reformas nos métodos de ensino para que se tornem mais adequados ao cenário atual da sociedade. Sua estrutura tem como objetivo permitir que o aluno desenvolva suas habilidades de trabalhar em grupo e encontrar soluções para um problema. Dessa forma, o foco é no desenvolvimento das capacidades dos alunos em vez de seguir uma estrutura de aulas expositivas como no ensino tradicional [de Souza e Luis Dourado 2015].

A aprendizagem colaborativa proporcionada pela ABP é um processo de mudança cultural no ensino superior, o professor tem um papel de tutor no espaço acadêmico, facilitando o aprendizado através da metodologia [Barrett and Moore 2010]. Nos componentes curriculares de Resolução de Problemas os alunos são organizados em equipes e devem trabalhar colaborativamente para encontrar soluções para problemas propostos pelos professores.

Cenários voltados para a pesquisa de WSN no contexto de disciplinas de Resolução de Problemas tornam os desafios citados anteriormente ainda mais aparentes. O ambiente acadêmico onde os recursos são escassos e os laboratórios não dispõem de equipamentos apropriados, apresentam barreiras para a prática de desenvolvimento com Wireless Sensor Networks. Considerando isso, a alternativa de utilizar simulações torna viável as práticas educativas em cenários que apresentem tais limitações.

O presente trabalho tem o objetivo de explorar a utilização de simulações de $W i$ reless Sensor Networks no contexto de Resolução de Problemas e está organizado da seguinte forma: na Seção 2 são discutidos os trabalhos relacionados, na Seção 3 é relatado um exemplo de simulação. A conclusão é apresentada na Seção 4 e, por fim, as referências.

\section{Trabalhos Relacionados}

A Tabela 1 apresenta os dez trabalhos relacionados a serem discutidos a seguir. Estes foram os estudos mais relevantes para a utilização como problemas base no desenvolvimento de componentes curriculares de Resolução de Problemas, encontrados no estudo de mapeamento sistemático realizado pelos autores do presente trabalho. 
Tabela 1. Trabalhos relacionados

\begin{tabular}{|c|l|}
\hline Referência & Título \\
\hline [Khelifi 2020] & $\begin{array}{l}\text { Monitoring System Based in Wireless Sensor } \\
\text { Network for Precision Agriculture }\end{array}$ \\
\hline [Shipu et al. 2018] & $\begin{array}{l}\text { Research on WSN Routing Algorithm for Ve- } \\
\text { getable Greenhouse }\end{array}$ \\
\hline [Jiang et al. 2018] & $\begin{array}{l}\text { Energy Efficient Smart Irrigation System Based } \\
\text { on 6LoWPAN }\end{array}$ \\
\hline [Zhang et al. 2019] & $\begin{array}{l}\text { Sensor-Cloud Based Precision Sprinkler Irriga- } \\
\text { tion Management System }\end{array}$ \\
\hline [Nandhini et al. 2017] & $\begin{array}{l}\text { Web Enabled Plant Disease Detection System } \\
\text { for Agricultural Applications Using WMSN }\end{array}$ \\
\hline [Linsner et al. 2019] & $\begin{array}{l}\text { Vulnerability Assessment in the Smart Farming } \\
\text { Infrastructure through Cyberattacks }\end{array}$ \\
\hline [Mahalakshmi et al. 2020] & IoT Sensor-Based Smart Agricultural System \\
\hline [Khan and Kumar 2019] & $\begin{array}{l}\text { Ambient Crop Field Monitoring for Improving } \\
\text { Context Based Agricultural by Mobile Sink in } \\
\text { WSN }\end{array}$ \\
\hline [Wang et al. 2019] & $\begin{array}{l}\text { LWS: A LoRaWAN Wireless Underground } \\
\text { Sensor Network Simulator for Agriculture Ap- } \\
\text { plications }\end{array}$ \\
\hline LWu et al. 2020] & $\begin{array}{l}\text { Long range wide area network for agricultural } \\
\text { wireless underground sensor networks }\end{array}$ \\
\hline
\end{tabular}

O primeiro trabalho a ser discutido é [Khelifi 2020], nele os autores propõem um algoritmo híbrido de rota no qual são utilizados clusters baseados em regiões para a implantação de sensores. O objetivo é, portanto, prover uma melhor cobertura para a área onde são utilizados os sensores.

A seguir, em [Shipu et al. 2018], os autores propõem o uso de algoritmos de roteamento para melhorar a comunicação e o consumo de energia em WSNs. Tal problema se mostra ideal para simular ambientes motivados para domínios de estufas de vegetais, uma vez que se encaixarem melhor no ambiente controlado.

Em [Jiang et al. 2018], os autores apresentam uma proposta que trata de um problema relevante para avaliar a eficiência em distâncias entre os nós da WSN. Por exemplo, em domínios de irrigação inteligente, onde os nós da rede necessitam de transmissão de longa distância e de baixo consumo de energia para operarem. Outro problema também derivado na motivação de irrigação é a tecnologia de nuvem de sensores vista em [Zhang et al. 2019].

Um problema comum investigado na literatura é para a detecção e alastramento de doenças. Por exemplo, [Nandhini et al. 2017] explora a simulação num sistema web de detecção de doenças para segmentar e classificar doenças em folhas de romã, tomate e berinjela com o uso de wireless multimedia sensor networks (WMSNs).

Outro problema possível de ser tratado em simulação é para a identificação de 
vulnerabilidades em WSNs. Por exemplo, o estudo de [Linsner et al. 2019] apresenta testes de ataques em WSNs, discutindo sobre questões de segurança na área e como elas afetam os custos de adesão. Outro trabalho neste problema adota um modelo de aplicação de encryption-as-a-service para verificar a comunicação entre nodos via criptografia [Mahalakshmi et al. 2020]. Ambos poderiam ser problemas utilizados em disciplinas de ABP com foco em segurança.

Outro problema para simulação envolve medir parâmetros pela mobilidade das bases de sensoriamento. Por exemplo, a solução proposta por [Khan and Kumar 2019] trata de um sistema de monitoramento de ambiente do campo utilizando uma base móvel para reduzir consumo de energia, delay, e hop count durante transmissão de dados.

Medir a proximidade da modelagem com cenários reais é outro problema possível de estudos exploratórios. Por exemplo, [Wang et al. 2019] e [Wu et al. 2020] trabalham no desenvolvimento de um simulador para investigar o uso de LoRaWan para WSNs subterrâneas com o objetivo de permitir uma modelagem mais próxima da implantação.

\section{Demonstração Conceitual}

Em nosso grupo de pesquisa temos como objetivo oferecer base para que alunos sejam aptos ao desenvolvimento de soluções para IoT em cenários de agricultura inteligente [Fialho et al. 2021]. Neste contexto, o emprego de sensores auxilia na monitoria de ambientes de produção agrícola por meio de computação aplicada para problemas que necessitem do processamento de sinais de diversos sensores espalhados nestes ambientes. Tipicamente em problemas de IoT, múltiplos sensores comunicam-se com um nodo central de processamento. O nodo central é capaz de acionar atuadores para controlar o ambiente de produção.

Buscando desenvolver estudos desta natureza, e que sejam voltados para a temática de Internet of Things, apresenta-se um estudo exploratório de emprego de simulação. Nossa motivação é fomentar uma base exploratória aplicada para a execução de futuras disciplinas de RP em Engenharia de Software. Estudos de simulação podem ser utilizados como provas de conceito práticas, e são comumente utilizados na indisponibilidade de hardware. Ou seja, podem ser alternativas interessantes para disciplinas de ABP sem a necessidade de se investir em hardware. Portanto, são fontes viáveis para se explorar em tais disciplinas.

Para desenvolver a simulação, utilizou-se a ferramenta Cooja Simulator, que é parte do ambiente Contiki ${ }^{1}$. A simulação foi desenvolvida utilizando o Cooja na versão 3.0 do Instant Contiki, a maquina virtual de Contiki OS disponibilizada por seus desenvolvedores. A escolha dessa ferramenta se deve a ser uma máquina virtual já configurada que simula o funcionamento do Contiki OS em um ambiente real. Os sensores utilizados para a simulação foram nodos Sky, que são um dos modelos disponíveis no simulador e podem ser vistos na Figura 1.

O ambiente simulado é composto por dez nodos sensores enviando dados para uma base. Na Figura 2 apresenta-se a topologia da simulação com o nodo 1 sendo a base e os de 2 a 11 sendo nodos sensores. A organização foi definida aleatoriamente pelo

\footnotetext{
${ }^{1}$ https://www.contiki-ng.org/
} 


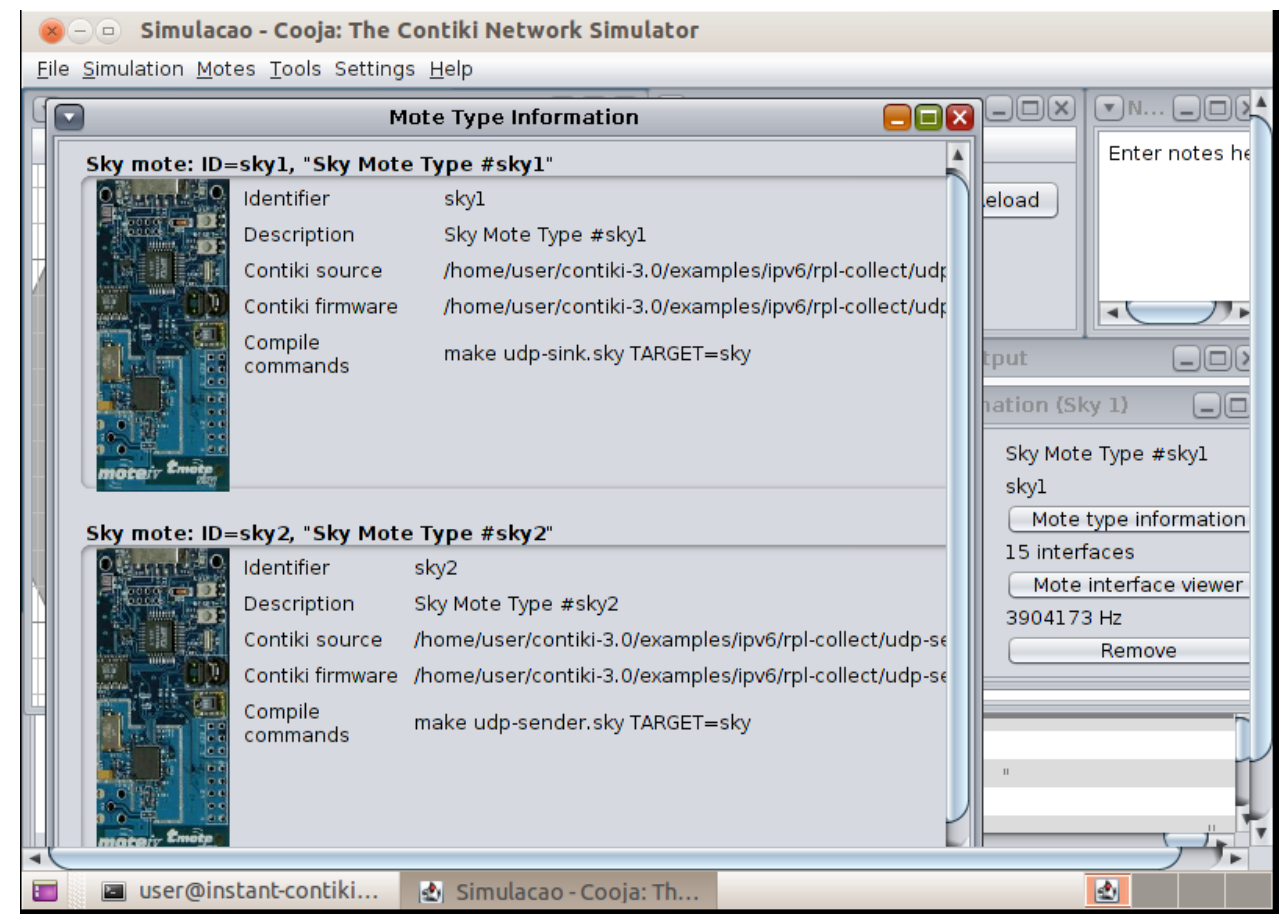

Figura 1. Sensores utilizados

simulador, no entanto foram feitos ajustes para que a base ficasse localizada no centro da grade com todos os nodos sensores dentro do seu alcance.

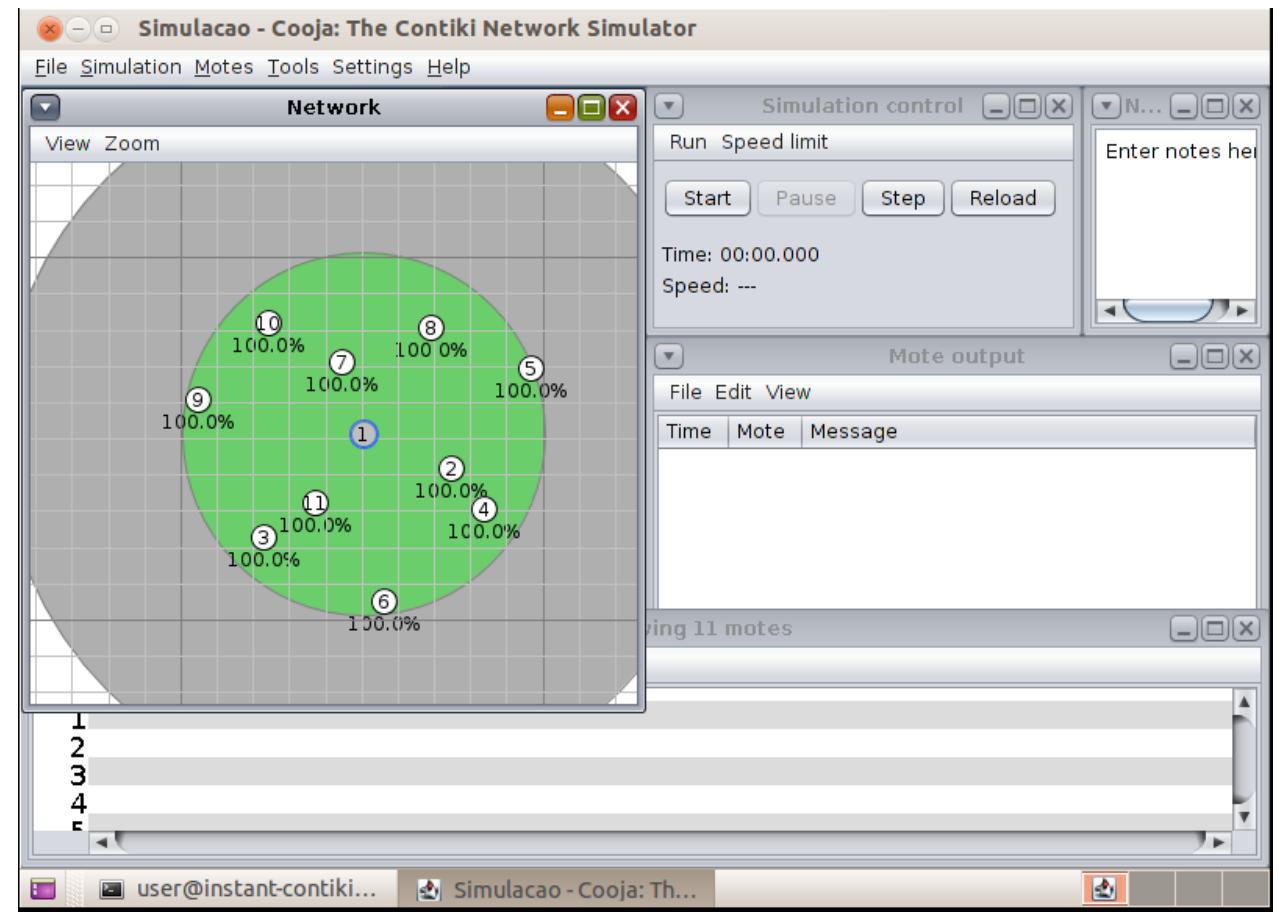

Figura 2. Topologia da simulação

Em um cenário utilizando um problema de WSN em aquaponia, onde hidroponia e aquicultura são combinados em um ambiente simbiótico, cada nodo sensor poderia repre- 
sentar um sensor real para diferentes parâmetros que precisam ser medidos no domínio. Em sua revisão, [Yanes et al. 2020], define 19 parâmetros considerados cruciais em sistemas aquapônicos. Estes parâmetros são descritos na Tabela 2.

\section{Tabela 2. Parâmetros Associados com Sistema Aquapônicos}

\begin{tabular}{|c|c|}
\hline Parâmetro & Descrição \\
\hline Água & $\begin{array}{l}\text { O fator mais importante em sistemas aquapônicos, afinal, é o meio usado para prover nutrientes para as plantas. } \\
\text { É também o fator mais complexo no que se trata de automação, pois depende do controle de diversos parâmetros } \\
\text { simultaneamente. }\end{array}$ \\
\hline Nitrificação & $\begin{array}{l}\text { É o processo de transformação da amônia-obtida a partir dos dejetos dos peixes-m nitrogênio com a utilização } \\
\text { de bactérias. Esse processo envolve o monitoramento do total de amônia-nitrogênio (TAN) presente na água e a } \\
\text { transformação da amônia em nitritos e nitratos pelas bactérias. Sempre monitorando para garantir que nenhuma } \\
\text { dessas substâncias alcance níveis tóxicos. }\end{array}$ \\
\hline $\mathrm{pH}$ & $\begin{array}{l}\text { É uma medida de concentração de íons de hidrogênio, comumente conhecido como uma medida de acidez ou } \\
\text { alcalinidade de uma solução. Alterações no pH da água podem alterar o equilíbrio do sistema aquapônico, alterando } \\
\text { a taxa de reprodução dos peixes ou causando deficiência de nutrientes nas plantas. }\end{array}$ \\
\hline Temperatura & $\begin{array}{l}\text { Está ligada à maioria dos outros parâmetros relacionados à água. Se a temperatura fica fora do intervalo ideal para os } \\
\text { organismos presentes no sistema aquapônico isso pode afetar o processo de nitrificação, pois afeta a produtividade } \\
\text { das bactérias, e também pode ser nocivo para a saúde dos peixes e das plantas. }\end{array}$ \\
\hline Nível & $\begin{array}{l}\text { importante principalmente para a parte de aquacultura do sistema. O nível de água em relação a quantidade de peixes } \\
\text { é o principal fator de estresse para os organismos, podendo afetar negativamente a sua saúde e crescimento. }\end{array}$ \\
\hline $\begin{array}{l}\text { Oxigênio dissol- } \\
\text { vido }\end{array}$ & $\begin{array}{l}\text { É a quantidade de oxigênio disponível na água para os organismos. Sendo reportado como o fator com efeitos mais } \\
\text { drásticos e imediatos em sistemas aquapônicos. O nível baixo de oxigênio dissolvido afeta a produção de TAN dos } \\
\text { peixes, o processo de nitrificação, pode fazer com que as raízes das plantas morram e facilita o surgimento de fungos. }\end{array}$ \\
\hline $\begin{array}{l}\text { Condutividade } \\
\text { elétrica }\end{array}$ & $\begin{array}{l}\text { é a medida da habilidade de um meio de conduzir corrente elétrica, quando se trata de água está altamente relacionada } \\
\text { à sua salinidade. Níveis baixos podem indicar que o sistema não está balanceado, enquanto níveis altos indicam que } \\
\text { a água está poluída—o que pode causar morte dos peixes. }\end{array}$ \\
\hline $\begin{array}{l}\text { Total de sólidos } \\
\text { dissolvidos }\end{array}$ & $\begin{array}{l}\text { Representam o conteúdo de sais inorgânicos, material orgânico e outros materiais dissolvidos na água. Níveis altos } \\
\text { podem tornar o ambiente tóxico para a maioria dos peixes. O sensor utilizado para o monitoramento costuma ser o } \\
\text { mesmo de condutividade elétrica. }\end{array}$ \\
\hline Salinidade & $\begin{array}{l}\text { Indica a concentração de sal presente na água e afeta o crescimento e densidade dos peixes. Também pode ser } \\
\text { monitorado com o mesmo sensor de condutividade elétrica. }\end{array}$ \\
\hline Dureza da água & $\begin{array}{l}\text { É a medida da concentração de sais de cálcio e magnésio com carga positiva presentes na água. Ambos são essenciais } \\
\text { para a saúde dos peixes, principalmente para a formação de escamas e ossos. Níveis baixos podem causar estresse } \\
\text { nos peixes e níveis altos podem ser letais, pois aumentam o pH da água, causando um baixo índice de nitrificação e } \\
\text { prejudicando a absorção de nutrientes pelas plantas. }\end{array}$ \\
\hline Alcalinidade & $\begin{array}{l}\text { É a medida de concentração das bases, normalmente de carbonato e bicarbonato. Diferente da dureza da água que } \\
\text { mede os íons positivos, a alcalinidade mede os íons negativos. Em níveis baixos faz com que pequenas quantidades } \\
\text { de ácidos alterem drasticamente o pH da água, enquanto níveis altos tornam amônia não tóxica em tóxica. }\end{array}$ \\
\hline Fluxo & $\begin{array}{l}\text { É extremamente importante para estimar a capacidade de filtração e nitrificação do sistema, além de determinar } \\
\text { a disponibilidade de nutrientes para as plantas. O fluxo ideal varia dependendo do tipo de sistema aquapônico } \\
\text { implementado. }\end{array}$ \\
\hline Ambiente & $\begin{array}{l}\text { Trata das condições do ar em contato com as plantas. Para garantir o crescimento estável e saudável dos peixes e das } \\
\text { plantas é necessário monitorar e controlar alguns fatores ambientais. }\end{array}$ \\
\hline $\begin{array}{l}\text { Temperatura do } \\
\text { ar }\end{array}$ & $\begin{array}{l}\text { Tem influência na saúde dos plantas, temperaturas altas fazem com que verduras comecem a florescer. Também é } \\
\text { responsável pela transpiração adequada das plantas. }\end{array}$ \\
\hline Umidade relativa & $\begin{array}{l}\text { É uma expressão da umidade do ar. A maioria das plantas produzidas em sistemas aquapônicos exigem que o ar seja } \\
\text { úmido, por isso é um parâmetro que deve ser bem gerenciado. }\end{array}$ \\
\hline $\begin{array}{l}\text { Dióxido de car- } \\
\text { bono }\end{array}$ & $\begin{array}{l}\text { É um componente essencial da fotossíntese, em ambientes de produção em massa é possível que as plantas utilizem } \\
\text { todo o dióxido de carbono do ambiente, fazendo necessário que se tenha o controle da disponibilidade e até mesmo } \\
\text { a adição de dióxido de carbono. }\end{array}$ \\
\hline $\begin{array}{l}\text { Umidade de subs- } \\
\text { trato }\end{array}$ & $\begin{array}{l}\text { Quando o componente hidropônico do sistema utiliza plantio em substrato é necessário monitorar a sua umidade. } \\
\text { Um sensor de umidade do solo oferece as leituras necessárias e em conjunto com as informaçoses sobre a quantidade } \\
\text { para o tipo de solo e planta pode-se facilmente manter a umidade dentro do ideal. }\end{array}$ \\
\hline $\begin{array}{l}\text { Intensidade de } \\
\text { luz }\end{array}$ & $\begin{array}{l}\text { É um fator essencial para a saúde das plantas, porém quando a produção se da em locais fechados pode se tornar } \\
\text { limitada ou inexistente. As plantas utilizam uma parte do espectro de luz chamada radiação fotossinteticamente } \\
\text { ativa. A presença de níveis adequados de luz garante o crescimento saudável das plantas. Em algumas culturas, } \\
\text { como alface e repolho, a presença de luz excessiva tem um efeito negativo no sabor das plantas. }\end{array}$ \\
\hline
\end{tabular}

Os códigos para o funcionamento da base e sensores utilizados foram exemplos disponíveis na ferramenta. A Figura 3 ilustra a execução da simulação, mostrando a comunicação entre nodos enquanto acontecem. Essas e outras informações podem ser capturadas durante a simulação para serem analisadas posteriormente na base 1 de processamento central do cenário de IoT. 


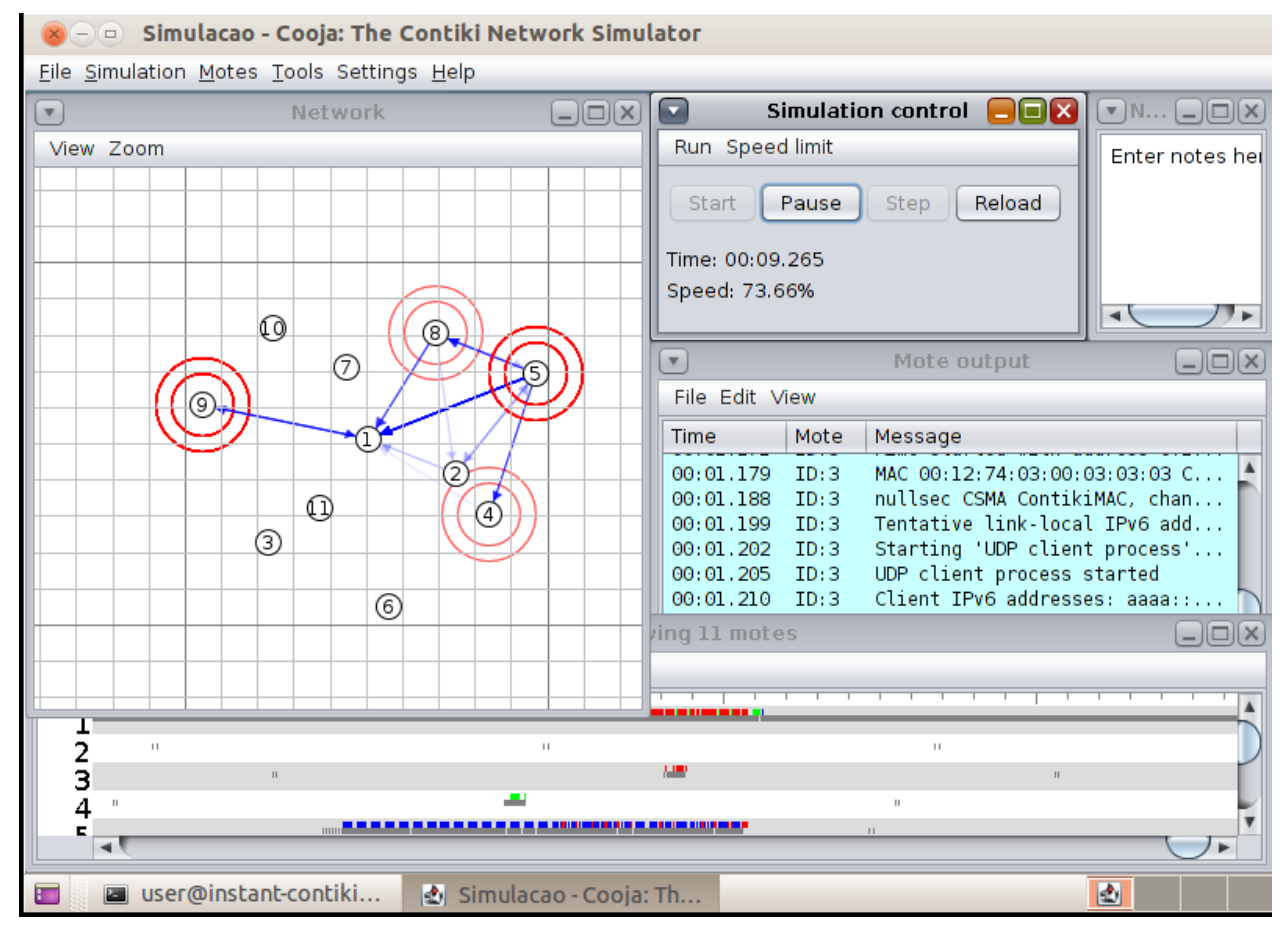

Figura 3. Simulação sendo executada

A Figura 4 apresenta um exemplo do tipo de informação que pode ser capturada durante a execução da simulação. Nesse exemplo o consumo médio de energia de todos os sensores é apresentado em um gráfico de barras com cores indicando o consumo para diferentes atividades que foram simuladas.

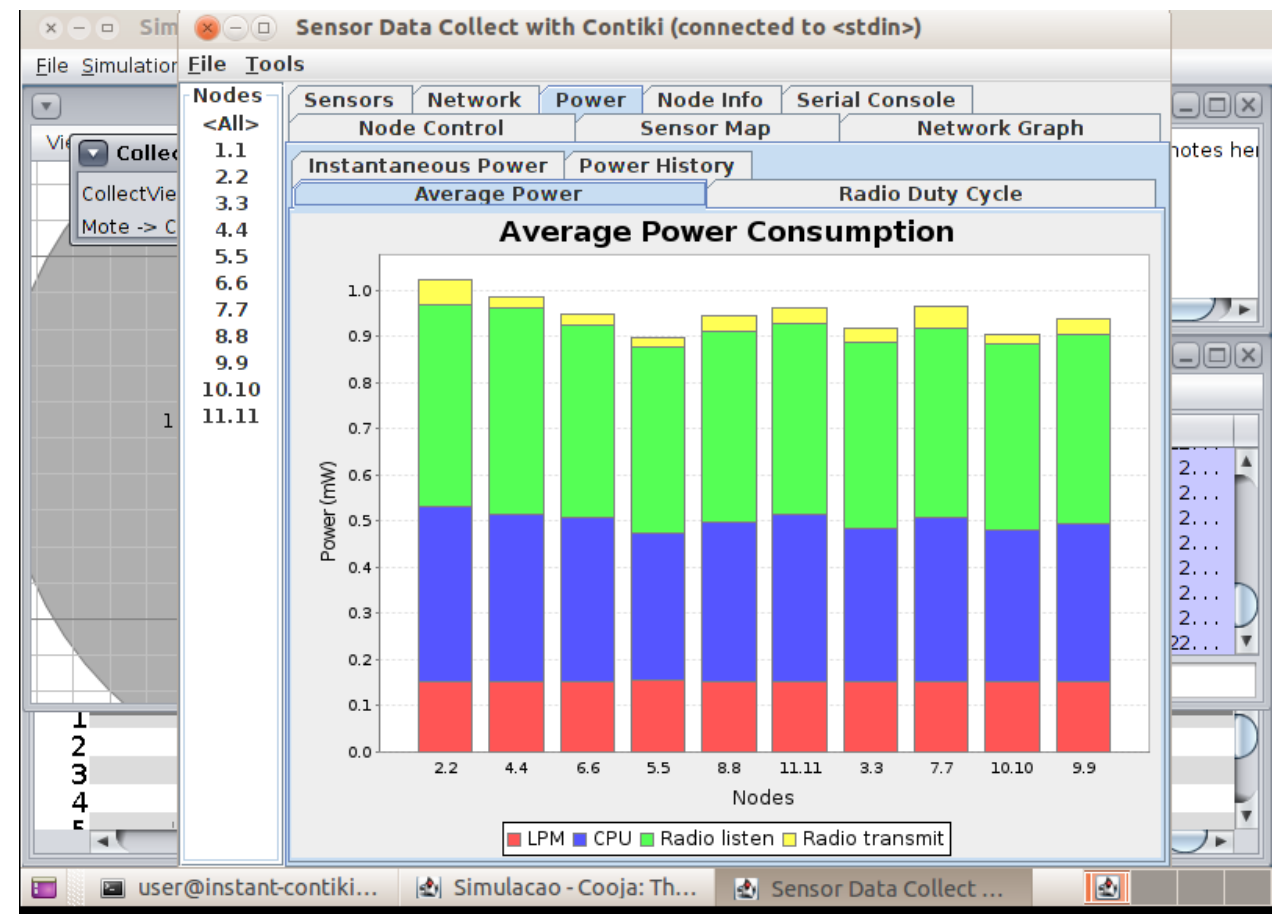

Figura 4. Consumo médio de energia 


\section{Conclusão}

A utilização de WSNs na agricultura tem se mostrado essencial para atender as demandas da área, porém a utilização de dispositivos físicos para a realização de testes se torna inviável pelo alto custo e impraticabilidade em alguns cenários educativos.

A literatura atual carece de estudos sobre o uso de simulações que caracterizem bem os problemas nos domínios de WSNs e agricultura, normalmente eles têm caráter mais científico, são desenhados com o intuito de testar as soluções propostas em simulação, e negligenciam demonstrações conceituais de um ambiente completo de sensoriamento. Ou seja, no geral, não são estudos prontos para utilização em disciplinas de ABP.

Esse trabalho buscou apresentar uma alternativa para o desenvolvimento de simulações em um ambiente acadêmico de uma forma simples para alunos e professores. Nosso objetivo foi demonstrar como simulações podem ser realizadas utilizando Cooja e oferecer algumas opções de áreas de pesquisa nas quais simulações podem ser aplicadas.

Nosso próximo objetivo é aplicar o estudo para problemas identificados em um estudo anterior de mapeamento sistemático, que levantou problemas do domínio de agricultura e respectivas soluções de simulação em IoT. Após definir cenários em que simulações possam ser usadas, o objetivo é desenvolver uma estratégia de ensino para ser utilizada por professores de disciplinas de ABP.

Portanto, o presente estudo apresentou uma pequena contribuição num estudo exploratório de um cenário que pretende-se utilizar em futuras disciplinas de Resolução de Problemas. A simulação apresentada utilizando o Cooja Simulator serve, portanto, de exemplo para a aplicação nesses componentes curriculares.

\section{Referências}

Abuarqoub, A., Hammoudeh, M., Alfayez, F., and Aldabbas, O. (2016). A Survey on Wireless Sensor Networks Simulation Tools and Testbeds, pages 283-302.

Barrett, T. and Moore, S. (2010). New approaches to problem-based learning: Revitalising your practice in higher education. Routledge.

de Souza e Luis Dourado, S. C. (2015). Aprendizagem baseada em problemas (abp): Um mÉtodo de aprendizagem inovador para o ensino educativo. HOLOS, 5(0):182-200.

Fialho, D., Basso, F., Luizelli, M., and Cabrera, S. (2021). Emprego de simulações computacionais em problemas envolvendo agricultura: Um estudo de mapeamento sistemático. In Anais do III Workshop em Modelagem e Simulação de Sistemas Intensivos em Software, pages 20-29, Porto Alegre, RS, Brasil. SBC.

Jiang, X., Yi, W., Chen, Y., and He, H. (2018). Energy Efficient Smart Irrigation System Based on 6LoWPAN: 4th International Conference, ICCCS 2018, Haikou, China, June 8-10, 2018, Revised Selected Papers, Part V, pages 308-319.

Khan, F. and Kumar, D. (2019). Ambient crop field monitoring for improving context based agricultural by mobile sink in wsn. Journal of Ambient Intelligence and Humanized Computing.

Khan, S., Pathan, A.-S. K., and Alrajeh, N. A. (2016). Wireless Sensor Networks: Current Status and Future Trends. CRC Press, Inc., Boca Raton, FL, USA, 1st edition. 
Khelifi, F. (2020). Monitoring System Based in Wireless Sensor Network for Precision Agriculture, pages 461-472. Springer International Publishing, Cham.

Linsner, S., Varma, R., and Reuter, C. (2019). Vulnerability assessment in the smart farming infrastructure through cyberattacks. In Meyer-Aurich, A., Gandorfer, M., Barta, N., Gronauer, A., Kantelhardt, J., and Floto, H., editors, 39. GIL-Jahrestagung, Digitalisierung für landwirtschaftliche Betriebe in kleinstrukturierten Regionen - ein Widerspruch in sich?, pages 119-124, Bonn. Gesellschaft für Informatik e.V.

Mahalakshmi, J., Kuppusamy, K., Kaleeswari, C., and Maheswari, P. (2020). Iot sensorbased smart agricultural system. In Subramanian, B., Chen, S.-S., and Reddy, K. R., editors, Emerging Technologies for Agriculture and Environment, pages 39-52, Singapore. Springer Singapore.

Nandhini, A., Rajendran, H., Sankararajan, R., and Indumathi, K. (2017). Web enabled plant disease detection system for agricultural applications using wmsn. Wireless Personal Communications.

ONU, N. U. (2021). Un calls for urgent action to feed the world's growing population healthily, equitably and sustainably.

Shipu, X., Yunsheng, W., Yong, L., Weixiong, R., Mingzhou, M., Jingyin, Z., and Chenxi, Z. (2018). Research on wsn routing algorithm for vegetable greenhouse. pages 37-42.

Thakur, D., Kumar, Y., Kumar, A., and Singh, P. K. (2019). Applicability of wireless sensor networks in precision agriculture: A review. Wireless Personal Communications, 107(1):471-512.

Wang, K. I.-K., Wu, S., Ivoghlian, A., Salcic, Z., Austin, A., and Zhou, X. (2019). Lws: A lorawan wireless underground sensor network simulator for agriculture applications. In 2019 IEEE (SmartWorld/SCALCOM/UIC/ATC/CBDCom/IOP/SCI), pages 475-482.

Wu, S., Austin, A. C., Ivoghlian, A., Bisht, A., Kevin, I., and Wang, K. (2020). Long range wide area network for agricultural wireless underground sensor networks. Journal of Ambient Intelligence and Humanized Computing, pages 1-17.

Yanes, A. R., Martinez, P., and Ahmad, R. (2020). Towards automated aquaponics: A review on monitoring, iot, and smart systems. Journal of Cleaner Production, 263:121571.

Zhang, M., Xiong, S., and Wang, L. (2019). Sensor-cloud based precision sprinkler irrigation management system. 